\title{
Etnografía del poder en la relación médico paciente, identificación de una forma de violencia simbólica hacia las mujeres en el Programa de Salud Reproductiva
}

\author{
Galaviz de Anda Jesús Martín, Maza Díaz-Cortés Octavio Martín,**
}

Carlos Alberto Prado Aguilar***

\section{- Resumen}

- En este trabajo se pretende estudiar la violencia de género en la

- relación médico paciente durante la procuración de servicios de salud

- reproductiva y hacer visible la violencia como problema de salud pú-

- blica, donde se están violentando los derechos sexuales y reproducti-

- vos de las mujeres. Es un estudio auto reflexivo sobre las conductas

- que se dan en la cotidianeidad de los médicos y sus pacientes. La

- dominación está presente en la relación médico-paciente, confor-

- mando un tipo de violencia de género, en la que se identifica el uso

- del poder en cuyo ejercicio el control recrea patrones de conducta de

- dominación-subordinación y la disposición de autonomía-no autono-

- mía, del médico hacia sus pacientes, condicionado por los esquemas

- de percepción, acción y pensamiento. Esta violencia genera un habi-

- tus con esquemas de acción, percepción y pensamiento, que le dan al

- médico una posición de dominante y al paciente una disposición de

- no autonomía dentro del campo de la medicina. El problema central

- es la violencia de género, mediante el uso de poder dentro de la

- relación médico-paciente, expresada simbólicamente en las mujeres

- usuarias del programa de salud reproductiva justificada desde el dis-

- curso médico por la finalidad disminuir la morbidez y la mortalidad

- de la mujer causado por el alto riesgo reproductivo a que se ven

- sometidas. LUX MÉDICA AÑO 8, NÚMERO 24, MAYO-AGOSTO 2013, PP 21-29

- Palabras clave: violencia de género, relación médico-paciente, - poder médico, salud reproductiva.

\section{Abstract}

This work aims to study the gender violence in the doctor-patient relationship during assistance of reproductive health services and to make visible the violence as a public health problem where they are violating the sexual and reproductive rights of women. It is a self-reflexive study about the behaviors that occur in a daily basis of physicians and their patients. The problem is present in the patientphysician relationship, forming a kind of gender violence, in which it identifies the use of power and control recreates behavioral patterns of domination and subordination and the provision of autonomy-not autonomy, the doctor to his patients, conditioned by the schemes of perception, action and thinking, this violence generates a habitus with action schemes, perception and thought, giving the doctor a dominant position and the patient a provision of no autonomy within the field of medicine. The central problem is the gender violence, using power in the doctor-patient relationship, symbolically expressed in women using reproductive health program justified by the medical discourse intended to reduce the morbidity and mortality of women caused by the high reproductive risk to which they are subjected. LUX MÉDICA AÑO 8, NÚMERO 24, MAYO-AGOSTO 2013, PP 21-29

Keywords: gender violence, doctor-patient relationship, medical power, reproductive health.

Profesor de tiempo completo, investigador del Departamento de Gineco-Obstetricia del Centro de Ciencias de la Salud de la Universidad Autónoma de Aguascalientes, Doctorante en Ciencias Sociales y Humanidades

** Doctor en Sociología, Investigador Titular C, SNI 1 Universidad Autónoma de Aguascalientes

*** Doctor en Salud Pública, Investigador Titular C Universidad Autónoma de Aguascalientes

Fecha de recibido: 24 de junio 2013

Fecha de aceptación: 23 de julio 2013

Correspondencia: Jesús Martín Galaviz de Anda, Departamento de Gineco-Obstetricia del Centro de Ciencias de la Salud de la Universidad Autónoma de Aguascalientes. Avenida Universidad \# 940, Edificio 107 planta alta, Ciudad Universitaria Aguascalientes, Ags. México CP 20131. Teléfono (449) 9108443, correo electrónico martingalaviz@yahoo.com 


\section{Introducción}

Este estudio es una aproximación a la violencia de género, derivada del uso de poder dentro de la interacción social de la relación médico-paciente, expresada simbólicamente, hacia las mujeres usuarias del Programa de Salud Reproductiva justificada por el discurso médico. La pregunta es, ¿cómo se expresa una relación médicopaciente que ejerce violencia de género en la procuración de la atención médica? La dominación de género puede estar presente en la relación médico-paciente, en la que se identifica el uso del poder del médico hacia sus pacientes, creando una relación asimétrica donde las mujeres se ven violentadas de manera simbólica.

El objetivo de la investigación es identificar si el uso del poder es un problema que trasciende como una más de las formas de la violencia de género de tipo simbólico, durante la entrevista, donde a las mujeres se les puede ejercer acciones de poder por parte del médico y se atenta en los derechos sexuales y reproductivos de las mujeres dentro del campo médico, constituyendo en este escenario, el de los consultorios médicos, una forma más de la violencia de género que hasta ahora no ha sido estudiada en Aguascalientes, México.

El reconocer la existencia de violencia en la relación médico-paciente, permitirá en el futuro que se pueda negociar las estrategias libres de violencia, así como la creación de políticas que liberen la presencia de violencia de género dentro de la práctica médica, aplicable en especial en las instituciones de seguridad social en México, que brindan servicios médicos de la salud reproductiva.

\section{| | | | | | | | | | | | | | | | | | | | | | | | | | | | | | | | | | | | | | | | | | | | | | | | | | | | | | | | | | | | | | | | | | | | | | | | | | | | | | | | | | | | | | | | | | | | | | | | | | |}

\section{Antecedentes}

La violencia en sus diferentes manifestaciones es un tema que nos trastoca a todos, no importa edad o género, pero se presenta en mayor medida en las mujeres. Esto se debe a que el género masculino está sostenido por patrones culturales ancestrales que están en relación con la fuerza, la agresión física y no mantenerse callados, sino imponer o hacer notar su presencia y poder, mientras la educación femenina está orientada hacia la subordinación y al no rebelarse. ${ }^{1}$

Estos patrones pueden ser llevados desde el ámbito privado del hogar, donde se aprenden y llevados a los espacios públicos, como son en los hospitales, lo cual explica porque estos patrones se replican en los ámbitos de la práctica médica.
La violencia es cultural y no es modificada de manera inmediata sino a través de reflexiones profundas, confrontaciones y experiencias personales que lleven a desvanecer las construcciones perversas del individuo. "La masculinidad es simultáneamente una posición dentro de las relaciones de género y es a su vez las prácticas por las cuales hombres y mujeres se comprometen en dicha posición y los efectos de estas prácticas en la experiencia corporal, en la personalidad del individuo y en la cultura" . ${ }^{2}$ Las diferentes formas de violencia se evidencian y estudian a partir de las investigaciones sobre estudios de género que han permitido identificarlas y vincularlas con pautas culturales sociales para hombres y mujeres. Podemos decir que la violencia de género abarca todos 
los actos mediante los cuales se discrimina, subestima, somete y subordina a las mujeres en los diferentes aspectos de su existencia. Es todo ataque material y simbólico que afecta su libertad, dignidad, intimidad e integridad moral y o física. . $^{3,4}$

La manifestación de las inconformidades por parte de las mujeres, ante los abusos o el no querer hacer ciertas tareas en el trabajo, lo hacen casi siempre de una manera callada, lo cual produce un mayor sufrimiento porque reprimen la ira. El no hablar y hacer explícita la inconformidad de algo puede ser el origen de un problema mucho mayor que traerá serias consecuencias como competencias y rivalidades entre las personas, o el generar ser recriminadas en el trato siguiente. Por esto, esta investigación concede la voz a las mujeres para que expresen como pacientes su inconformidad ante el trato con violencia simbólica dentro del espacio médico. ${ }^{5}$

Definir la violencia contra las mujeres en el ámbito médico, implica describir una multiplicidad de actos, hechos u omisiones que las dañan y perjudican en los diversos aspectos de sus vidas, representado violaciones a sus derechos humanos, a sus derechos sexuales y reproductivos. ${ }^{6}$ Existe, además, una discriminación basada en el sexo, consolidada en pautas culturales de sumisión y que en el devenir histórico ha producido la "naturalización" de la violencia de género. 7,8 Estas pautas se reproducen en el ámbito médico. La forma del cuerpo asociada con la significación como sana, enferma, hipertensa, gestante, diabética, "abortadora", parturienta etc.; y además termina siendo considerada como objeto. La agresión a los cuerpos puede parecer en algún momento como una parte del trato hacia las pacientes cuando pueden ser actos violentos que son tan cotidianos que se consideraran naturales (no grite, no llore, puje, suba, baje, acomódese, desnúdese, muestre su cuerpo, etc. sin explicarles la razón de estas precisiones, ni hacerlo en el tono amable que no parezca un imperativo y que además hace sentir como un acto forzado a las pacientes); hecho que las hace más vulnerables y favorece que las mujeres sean las destinatarias de violencias estructurales y coyunturales. ${ }^{8}$

En el imaginario social de los consultorios médicos se han construido prejuicios en torno a las mujeres, que se comportan como pacientes cooperadoras o no cooperadoras, destinándoseles las etiquetas por parte de los médicos. ${ }^{7,9}$

El desafío es hacer un ejercicio de análisis del contexto de vida de las mujeres, percibiendo que la violencia con sesgo de género está presente en fenómenos tan diversos, los más representativos en la vida de las instituciones de salud como: coerción para el uso de anticonceptivos, coerción para la participación en pruebas clínicas, discriminación en salud, irrespeto al consentimiento informado, maltrato $y$ mala calidad de atención en los servicios de salud, negativa de proveer servicios de salud sexual y reproductiva, entre otros. ${ }^{10,11}$

Es claro que en las relaciones de médicos y pacientes no existe el poder simétrico, aunque esto pareciera como el estado ideal para el ejercicio de poder entre ellos, como encaja en la definición de la violencia ejercida hacia la mujer como "cualquier acción o conducta basada en su género, que cause la muerte, daño, o sufrimiento físico, sexual psicológico de la mujer, tanto en el ámbito público como en el privado". 12,13

La violencia de género, aterrizada en el contexto de la relación médico-paciente es aquella que lastima a un individuo (hombre ó mujer) dentro del espacio social, como el espacio médico y se presenta frecuentemente, a través del lenguaje hablado y no hablado. Para los fines de esta investigación se entiende por ambiente-espacio o campo médico, a la construcción subjetiva de un espacio de interacción como el lugar donde el individuo-médico desarrolla actividades que se relacionan con aspectos la- 
borales y académicos o que implican relaciones sociales que median en una relación médico-paciente. ${ }^{14}$

Las manifestaciones de la violencia en los espacios médicos son cada vez más evidentes, pues están acompañadas de comportamientos que no siempre pueden ser explicados, los conflictos y las tensiones que emanan adquieren características mucho más complejas, lo cual incentiva a la investigación actual, motivo por el cual ha llamado la atención para justificar a realizar esta investigación. ${ }^{14-17}$

\section{| | | | | | | | | | | | | | | | | | | | | | | | | | | | | | | | | | | | | | | | | | | | | | | | | | | | | | | | | | | | | | | | | | | | | | | | | | | | | | | | | | | | | | | | | | | | | | | | | | | | |}

\section{Metodología}

A partir de la pregunta, sobre la existencia de violencia de género en la relación médico- paciente en la consulta externa de ginecología, dentro del sistema de seguridad social, se decidió utilizar la metodología cualitativa. Con los métodos cualitativos de entrevista profunda, análisis del discurso y de observación participante, se ha podido interpretar y explicar la percepción de los actores sociales del uso de poder, además de identificar los símbolos que refuerzan la violencia de género de tipo simbólico mediante el interaccionismo simbólico y la interacción cara a cara, y el análisis de la conducta que ofrecen los médicos bajo la interpretación sociológica del problema médico. ${ }^{18,19}$

Se buscó el apoyo de dos entrevistadores con experiencia. Se diseñó una entrevista abierta con preguntas abiertas tanto a las pacientes como a los médicos, para tratar de entender cuál es la apreciación que tienen acerca de la relación médico paciente. Los informantes se obtuvieron por el catálogo de médicos prestadores del servicio de atención a mujeres. Los escenarios sociales donde se llevaron a cabo las interacciones entre los médicos y las pacientes fueron el consultorio de Ginecología en el área de consulta externa del hospital de la Seguridad Social, así como el área física destinada para el Jefe de Ginecología que atiende a las pacientes que tienen algún conflicto de atención con alguno de los médicos. Se realizó la observación del participante dentro de los escenarios del campo médico. ${ }^{20}$ Se optó por la entrevista no estructurada para dar la oportunidad de conocer la realidad de los actores sociales desde sus propias apreciaciones y su propio lenguaje. Una vez iniciadas las primeras entrevistas piloto se determinó el tipo de pacientes y médicos con que nos encontraríamos. Se entrevistaron a las autoridades competentes, médicos y pacientes usuarias del programa de Salud Reproductiva.

\section{Selección de los informantes}

Para la elección de informantes, fueron escogidos deliberadamente de acuerdo a quienes presentaron características especiales, de acuerdo a los criterios y atributos establecidos por los objetivos de la investigación. El tipo de informante para el caso crítico fueron los sujetos conocedores de lo típico, normal y promedio.

Se seleccionó al grupo de pacientes 1, porque es ahí donde hay más quejas de la atención medica y porque al estar cautivos los médicos y pacientes a una cartera fija y así ser atendidas un grupo de pacientes por el mismo médico, es fácil identificar los puntos de conflicto de la relación médicopaciente y por su naturaleza de la dinámica de estar en un área que permita identificarlas y entrevistarlas sin causar problemas de interferir con su padecimiento, como es el caso del grupo de pacientes tipo 2 que van buscando la atención médica en calidad de urgente como por ejemplo: hemorragia, o trabajo de parto, y se entiende no es el momento adecuado y ni tampoco nos ofrecen 
el interés que se pretende en esta investigación. Además que tampoco tendrán un médico fijo en particular sino que siempre habrá un médico diferente en cada turno y en cada día de la semana, como también es el caso de pacientes del grupo 3. (Véase la tipología de las pacientes en resultados)

Uno de los atributos que debió tener el informante fue la buena voluntad de dar información veraz y que además tuviera la capacidad suficiente de trasmitir la información al investigador. El tratamiento de los datos se realizó en tres etapas: el planteamiento de la entrevista, registro, trascripción y elaboración de las entrevistas y finalmente su análisis e interpretación. De manera complementaria se utilizaron los relatos solicitados, el cuaderno, bitácora, documentos personales, grabaciones de audio y una guía flexible de entrevista.

| | | | | | | | | | | | | | | | | | | | | | | | | | | | | | | | | | | | | | | | | | | | | | | | | | | | | | | | | | | | | | | | | | | | | | | | | | | | | | | | | | | | | | | | | | | | | | | | | | | | | | | | | | | |

\section{Resultados}

\section{Tipología de médicos}

Encontramos un solo tipo en los médicos que resultó de características muy homogéneo. Todos refieren haber sido capacitados en bioética bajo la corriente del principialismo, son todos médicos de base del sistema de contratación dentro de la institución y pertenecen a la ideología del contrato médico social de tipo explícito, (un contrato colectivo de trabajo, bajo la anuencia de la parte institucional como sindical) y reconocen estar bajo estas características.

\section{Tipología de las pacientes}

Por sus características se pudo agrupar a las pacientes en tres tipos.

1. Las pacientes tipo 1: son las que acuden citadas al hospital con una fecha y hora y con un ginecólogo en específico. Su problemática de salud está relacionada con el programa de Salud Reproductiva. Acuden al área de consulta externa y siguen una trayectoria bien definida dentro del hospital.

2. Las pacientes de tipo 2: son las que acuden sin cita, a cualquier hora del día en todos los días del año con un pro- blema de fondo de carácter de Urgencia Médica y que van en específico al servicio de Urgencias de Ginecología y Obstetricia. La dinámica de atención es rápida, por un personal en un servicio aparte muy localizado estratégicamente que facilita su pronta atención y éste se conecta a su vez hacia otras áreas de atención como pueden ser laboratorio, quirófanos, etc.

3. Las pacientes de tipo 3: son las que están dentro del área de hospitalización. Por la naturaleza heterogénea de sus edades, padecimientos y motivos de estar en el hospital pueden estar incluidas pacientes de diversos programas de atención médica, no solo las del programa de Salud Reproductiva. Aquí los médicos que las atienden no son fijos, sino más bien los que van rolando según la guardia de cada turno y de cada día de la semana.

En cuanto a la relación médico paciente

- Aspectos de la bioética: todos los médicos recibieron un curso sobre la corriente bioética del principialismo y todos se perciben que actúan bajo los principios de la beneficencia, la no maleficencia, respeto por la autonomía y la justicia para ejercer la práctica médica. Al ser interrogados sobre si cometen actos de 
violencia de género en su práctica médica, todos niegan percibir estas conductas en las propias actuaciones, todos desconocen el concepto de violencia de género de tipo institucional.

- De la deontología: todos los médicos afirman estar dentro de la normativa vigente sobre la práctica de salud reproductiva, no perciben actuar fuera de ella y se siente licitados en un buen actuar.

- Contrato social del médico de tipo implícito y explícito: al ser interrogados sobre qué es el contrato social del médico se refieren solo a lo que es la parte deontológica y se mantienen al margen de las órdenes que se reciben de la jefatura y sentir además que su práctica médica está protegida por el órgano sindical.

- De la dinámica institucional de sobrecarga de trabajo y sindicalismos: la mayoría percibe que están en continuo conflicto con las autoridades y con las pacientes debido a estar sometidos a exceso de trabajo, lo cual les justifica que la atención médica esté en detrimento para las pacientes y en desgaste físico y emocional para los médicos, y en esta parte coinciden además las opiniones de los médicos que se sienten desprotegidos del aparato jurídico institucional ante una situación médico legal, aun siendo las pacientes una "pertenencia" de la institución.

- De los antecedentes de los médicos y su aspiración a ser médicos: dicen los médicos que en principio ellos quieren hacer el bien y no buscan hacer mal a los pacientes y que desde sus orígenes en las escuela de medicina tiene un ideal de ser buenos médicos
Sobre su naturaleza de ser buenos médicos y la contaminación dentro de la institución con pautas de conducta homogeneizadoras al maltrato del paciente: se observa que los médicos más jóvenes llevan conductas más empáticas en su atención con las pacientes y conforme los médicos son de más edad, suelen referir expresiones de fastidio al ser entrevistados y referirse a estar cansados de la institución y de sus pacientes y se justifican con expresiones como "no me toca, ya me tienen cansado con tantas pacientes, y los jefes nos tienen hasta el tope", al ser interrogados algunos médicos recuerdan haber sido muy buena onda y que conforme pasan los años el cansancio los ha hecho caer en el fastidio cotidiano y usan una frase que expresa a manera de justificación sobre el porqué están así diciendo que "si los otros médicos no hacen su trabajo, porqué ellos sí lo han de hacer... luego les harán hacer más trabajo si terminan pronto de hacer lo suyo", "entre más haces, más te friegan"

- El médico que no se percibe violento: ningún médico se percibe violento de ninguna manera con sus pacientes, aunque dicen que sí hay otros médicos que sí lo son, pero se niegan a citar ejemplos o citar a quienes se refieren con cierta sonrisa de malicia en sus rostros.

- Los resultados de las percepciones de las pacientes: Todas las quejas que reportaron las pacientes fueron sobre el tiempo. Todas las pacientes entrevistadas, refirieron estar muy molestas y que se sentían violentadas por la espera de tanto "tiempo" de espera para ser atendidas, así como el "poco tiempo" que se les dedica durante la entrevista. Algunas refirieron especialmente que poder tener cita con el jefe del servi- 
cio es más de una semana porque este personaje "no tiene tiempo" de atenderlas, lo cual les ha generado mucho malestar y eso lo llaman ellas como una falta de respeto y lo cualifican como un trato violento. Por la observación participante se percibió que las mayoría de las pacientes reciben un trato que se podría interpretar como impersonal al no recibir un saludo amable al llegar la paciente o al salir, al solo recibir expresiones imperativas que ordenan a la paciente por parte del médico y la enfermera a que haga tales acciones como desvestirse, o subirse, pararse, al ser conducidas en el ritual de la consulta médica y que al ser interrogadas estas pacientes, ninguna percibió que este fuera un mal trato y que esto no es un trato violento hacia ellas de parte del médico ó de la enfermera, algunas has- ta justifican estas acciones con expresiones como "pos es que son médicos" como licitando que en el orden natural de las cosas el ser médico da posición para dar ese trato.

La observación de los procesos de salud reproductiva: demostró que los tiempos de espera en la procuración del la salpingoclasia post evento obstétrico puede tardar entre 12 horas a 3 días o ser reprogramadas hasta seis semanas vía consulta externa y en el caso de las vasectomías son programados los varones en una semana y su meta de cumplimiento es del $100 \%$, lo cual puede ser causa de embarazos no deseados, deserciones de uso de métodos de planificación familiar y en los análisis de la mortalidad materna de un año se observó la falta de un método de control de la fertilidad en 4 de 6 pacientes del 2011.

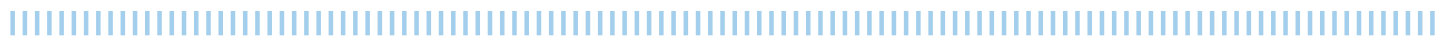

\section{Discusión}

Los médicos actúan de buena fe y no tienen la intención de ser violentos en el desempeño de sus funciones, no hay tal percepción por parte de ellos, mas sí perciben sentirse violentados por el sistema institucional al tener sobrecarga de trabajo y estar cansados y generar apatía ante su trabajo conforme van pasando años de laborar dentro de la institución, comparado con los médicos de reciente ingreso que suelen estar más empáticos con sus pacientes, todos siente una desconfianza del sistema jurídico institucional que los hace percibirse sin protección legal. Todos están conscientes de actuar bajo normas bioéticas de la corriente del principialismo y manejarse dentro del contexto del contrato médico explícito de su contrato colectivo de trabajo.

Las pacientes no perciben en el trato de sus médicos la violencia, y parece que son ellas mismas quienes lo justifican por efecto quizás del imaginario colectivo en relación al trato esperado que pueden recibir del médico dentro de la institución y lo legitimasen tomando como tácito y normal. El problema que perciben como fundamental es el factor tiempo de espera muy larga en recibir una atención y el tiempo muy corto en que reciben la intervención médica que les resulta insuficiente. Por su parte los médicos dicen tener poco tiempo para dedicarles a sus pacientes. Los procesos de intervención en otorgar atención de salpingoclasia a las mujeres es muy largo porque así está diseñado el sistema de atención de salpingoclasia post evento obstétrico y en cambio en de vasectomía sin bisturí es eficiente hasta el $100 \%$.

\section{Consideraciones finales}

Algunos aspectos de la salud de la mujer han sido considerados prioritarios por el estado mexicano desde hace varias décadas, 
especialmente la anticoncepción y la salud materno-infantil. En años más recientes, la preocupación se ha extendido a otras áreas de la vida de la mujer. Ejemplos de ello son la participación activa y decidida de México en las conferencias convocadas por Naciones Unidas en Cairo (ICPD, 1994) y Beijing (ICW, 1995) y las reuniones internacionales de seguimiento cinco años después, la ley contra la violencia doméstica promulgada en años recientes, la creación del Programa Nacional de la Mujer durante la administración anterior. ${ }^{20}$

Pese a estos esfuerzos, los avances son aún limitados. En primer lugar, aún se restringe esencialmente el concepto de salud de la mujer a sus funciones reproductivas y no se ha incorporado un enfoque de ciclo vital, imprescindible para ofrecer una respuesta efectiva a las necesidades integrales de salud de la mujer. Es así como poco se sabe de las necesidades especiales de las niñas o de la frecuencia y las características (determinadas biológica y socialmente) que los problemas de salud que no son exclusivos de las mujeres adoptan en ellas, ni de cómo ofrecer una atención que responda efectivamente a estas necesidades.

A la falta de conciencia y de información contribuye el hecho que las estadísticas hospitalarias y de vigilancia epidemiológica no están desagregadas por sexo. Si bien la cobertura de servicios para problemas de salud reproductiva y en general los de morbilidad y de seguimiento epidemiológico, es razonablemente amplia, la calidad técni$\mathrm{ca}$, humana y ética sigue siendo en ocasiones irregular y deficiente, ejemplos de ello son la injustificadamente alta prevalencia de cáncer de cuello uterino, para el que existen pruebas de detección, diagnóstico y tratamiento eficientes; el mal trato que frecuentemente reciben las mujeres en los servicios de atención obstétrica, especialmente cuando acuden a las instituciones para recibir cuidados para complicaciones del aborto, y las violaciones a los derechos reproductivos y al consentimiento informado que manifiestan las usuarias de los servicios de planificación familiar. ${ }^{21}$

El empoderamiento es un proceso que permite la conceptualización de una posibilidad de vida de acuerdo a las necesidades y a las decisiones del empoderado. Es un trayecto lento y continuo que acrecienta el poder y control sobre sus vidas en la toma de decisiones y que ayuda en la construcción de la autoconfianza, la ampliación de opciones y oportunidades al creciente acceso y control de los recursos. ${ }^{22,23} \mathrm{Si}$ cada sociedad adopta e impone cada normalidad para sus individuos, ya sea asimetría de los sexos en forma horizontal o relaciones de verticalidad de dominación y subordinación, son el resultado de construcciones sociales que se adoptan como naturales y se les nombra como normal por el uso y la costumbre de sus seres sociales. ${ }^{15}$

Finalmente, vale la pena enfatizar, que a pesar de la obvia importancia de tomar al género como una variable esencial en la determinación de la salud y enfermedad, en el sector público el término "género" es, hasta ahora, parte de la retórica, pero no se ha incorporado todavía a las políticas y programas de un modo consistente y articulado. Por otro lado, con objeto de atender los rezagos en la calidad técnica, humana y ética que sigue siendo irregular y deficiente en los problemas de salud reproductiva, y dada la importancia de incorporar la perspectiva de género en el sector salud y promover el diseño programas de información, investigación y atención de la salud incluyendo metodologías cualitativas de investigación en salud. ${ }^{24}$ 


\section{Conclusiones}

El presente estudio arroja como conocimiento nuevo, la tipificación de violencia de género del tipo institucional, con la variante o subtipo de la no intervención a tiempo en la procuración de servicios de salud reproductiva. En el investigador deja la transversalización de las disciplinas: el ser los mismos actores sociales que participan en los servicios de Salud Reproductiva y será observadores participantes y analizadores de los fenómenos sociales y médicos, puede ser una gran contribución si se fomenta el desarrollo de la Teoría Fundamentada para que se entienda mejor la problemática partiendo desde la base empírica y desarrollar teorías sociológicas que expliquen los fenómenos del conflicto médico desde una perspectiva social que contribuyan en mejorar los programas de salud. Por último, se evidencia la necesidad de profundizar en Teoría Fundamentada para estudios ulteriores bajo la capacitación del personal de salud que guste de profundizar en las ciencias sociales como una herramienta científica que ayude a explicar fenómenos sociales en los ámbitos médicos y que puedan crear desde ahí las políticas públicas que mejoren la equidad del género.

\section{Bibliografía}

1 Bourdieu, Pierre, la Sociología de la cultura de Pierre Bourdieu, éd. Grijalbo pp.9- 50. (1990)

2 Connell, R. W. and James W. 1995; 71 Hegemonic Masculinity: Rethinking the Concept Gender Society 2005; $19 ; 829$.

3 Roberto Castro, Carmen Castro, De Pacientes a Exigentes, 2010, Pág. 17.

4 Castro R. y Bronfman M. Teoría feminista y sociología médica: Bases para una discusión. En: Figueroa Perea JG. La condición de la mujer en el espacio de la salud. El Colegio de México, México. 1998.

5 Alberoni, Francesco (1991), Los envidiosos, Gedisa Editorial, España.

6 Montesinos, Rafael (2002) Las rutas de la masculinidad, Gedisa, España.

7 Castro R. y Bronfman M. Teoría feminista y sociología médica: Bases para una discusión. En: Figueroa Perea JG. La condición de la mujer en el espacio de la salud. El Colegio de México, México. 1998.

8 Las Luchas Clasificatorias en torno al Aborto, Estudios Sociológicos XXIV: 72,2006-Pág. 638.

9 Velásquez, Susana (2006) Violencias Cotidianas, violencia de género, Paidós, Buenos Aires.

10 Heise, Pitanguy y Germain, Violencia contra la mujer: La carga oculta de salud. Washington, D.C., OPS, 1994

11 Gender, Women and Health in the Americas. PAHO. Scientific Publicación no.541Gómez E. (Editora). Washington, D.C. 1993.

12 Maza, Octavio. (2009) El Empoderamiento de las Mujeres. Una revisión de distintas formas de trabajo no asalariado. Instituto Aguascalentense de las mujeres; Aguascalientes, México.

13 Maza, Octavio. (2009) El Empoderamiento de las
Mujeres. Una revisión de distintas formas de trabajo no asalariado. Instituto Aguascalentense de las mujeres; Aguascalientes, México pp. 23.

14 Rodríguez, Josep, Sociología de los ambulatorios, Análisis de la asistencia sanitaria primaria de la Seguridad Social, Ariel, Barcelona, 1979, Págs. 95-102

15 Foucault, Michel, Microfísica del Poder Ediciones de la Pinqueta, 1980 pp.153-162.

16 Gómez Gómez (Editora) Género, Mujer y Salud en las Américas. Washington D.C.: Organización

17 George Ritzer, Teoría Sociológica Contemporánea, Madrid McGraw-Hill, 1993. Pág.239

18 Rodríguez Gómez, Gregorio et al., Selección de informantes y recogida de datos

19 Wallace, WL. La Lógica de la Ciencia en Sociología. Cap. 1 Introducción. Pág. 29.

20 ONU. Ban Ki-Moon Objetivos de Desarrollo del Milenio. Informe 2008. Objetivo 5. Mejorar la salud materna. 24-27.

21 Szasz I. Género y Salud. "Propuesta para el análisis de una relación compleja". En: Bronfman M y Castro R: "Salud, Cambio social y políticas: Perspectivas desde América Latina. México. EDAMEX, 1999; P: 109-118 México, EDAMEX, 1999.

22 Maza, Octavio. (2009) El Empoderamiento de las Mujeres. Una revisión de distintas formas de trabajo no asalariado. Instituto Aguascalentense de las mujeres; Aguascalientes, México pp. 5.

23 "Sobre el inexistente pero insustituible término "empoderamiento". Población. Boletín Num. 8 The John D. and Catherine T. MacArthur Foundation. Programa de Seguridad y Sustentabilidad. Noviembre, 1999. P. 24

24 Taylor, S.J. y Bogdan, R. 1987, La observación participante en el campo, en introducción a los métodos cualitativos de investigación, Barcelona, Paidos, p 50-99 